\title{
A Gente Não Quer Só Comer: uma Abordagem de Marketing Social para a Alimentação Saudável
}

\author{
Edilaine Samara Pascoal de Oliveira \\ Universidade Federal da Paraíba - UFPB - Brasil \\ edilainepascoal25@gmail.com \\ ORCID: 0000-0002-3132-0571 \\ Stephanie Ingrid Souza Barboza \\ Universidade Federal da Paraíba - UFPB - Brasil \\ stephanieisb@gmail.com \\ ORCID: 0000-0002-2640-2070
}

We Do Not Want to Just Eat: a Social Marketing Approach to Healthy Eating

Submetido em 29/03/2019; Aprovado em 31/10/2019.

\section{RESUMO}

Objetivo: 0 objetivo desse estudo foi analisar a percepção dos agentes dos níveis upstream e midstream do modelo ecológico de marketing social sobre o consumo alimentar dos jovens, adotando uma abordagem qualitativa de caráter exploratório. Método: Para a coleta de dados, foram realizadas entrevistas baseadas em um roteiro semiestruturado dividido em três dimensões: questões sobre as barreiras para uma alimentação saudável, a regulação alimentar e a consciência sobre saúde. A pesquisa foi realizada com dez sujeitos, sendo cinco secretários municipais de saúde (agentes upstream) e cinco nutricionistas vinculados ao setor público (agentes midstream). As entrevistas foram analisadas a partir da análise de conteúdo. Resultados: Os resultados indicaram que as principais barreiras para adoção de uma alimentação saudável se relacionam com: a questão midiática, os aspectos culturais e o fator tempo aliado ao acesso de alimentos não saudáveis. Implicações práticas e teóricas: Os agentes consideram necessário que haja uma conscientização, fazendo uso de ferramentas de marketing social apoiada pelo governo, com intuito de implantar ações para a reeducação alimentar e prevenção de doenças, além de explorar os benefícios e incentivos para a alimentação saudável.

Palavras-Chave: Alimentação saudável; Adolescentes; Marketing Social.

\section{ABSTRACT}

Objective: The objective of this study was to analyze the perception of the agents of the upstream and midstream levels of the ecological model of social marketing on the food consumption of young people, adopting a qualitative exploratory approach. Method: For data collection, interviews were conducted based on a semi-structured script divided into three dimensions, involving questions about the barriers to healthy eating, food regulation and health awareness. The research was carried out with ten subjects, being five municipal health secretaries (upstream agents) and five nutritionists linked to the public sector (midstream agents). The interviews were analyzed from the content analysis. Results:The results indicated that the main barriers to adoption of healthy food relate to: the mediatic issue, cultural aspects, and the time factor allied to the access of unhealthy foods. Practical and theoretical implications: Agents consider it necessary to raise awareness by making use of government-supported social marketing tools to implement actions for food re-education and disease prevention, as well as to explore the benefits and incentives for healthy eating.

Keywords: Healthy eating; Adolescents; Social Marketing.

\section{Introdução}

Muitos são os problemas de saúde relacionados à alimentação, os mais evidentes são relativos às Doenças Crônicas Não Transmissíveis (DCNT), responsáveis por aproximadamente $70 \%$ de todas as mortes no mundo (Malta et al., 2017). De maneira geral, tal fato tem repercutido com mais força entre 
os mais jovens. Dados indicam que houve um aumento de mais de dez vezes no número de crianças e adolescentes obesos com idade entre 5 e 19 anos, nas últimas quatro décadas, um número alarmante que passou de 11 milhões em 1975 para 124 milhões em 2016 (Abarca-Gomez, 2017). Globalmente, a prevalência de obesidade entre crianças e adolescentes de 5 a 19 anos aumentou de 0,8\% em 1975 para 6,8\% em 2016.

Considerando que os hábitos alimentares inadequados na adolescência constituem fatores de risco para a obesidade e outras doenças crônicas não transmissíveis na vida adulta, é necessário dar a devida importância ao estímulo precoce no que concerne ao desenvolvimento de hábitos saudáveis nos indivíduos, buscando alertar o consumo excessivo de alimentos processados, os quais se relacionam com esses problemas (Alberga et al., 2012; Madruga et al., 2012). Ciente que as complicações dessas doenças também são responsáveis pela redução da qualidade de vida e aumento de outras morbidades, a importância de uma abordagem alimentar para o controle, a prevenção e o acompanhamento dessas afecções torna-se relevante. A Organização Mundial da Saúde propôs um incentivo nas intervenções políticas e uma regulação alimentar mais efetiva, com medidas regulatórias nacionais sobre nutrição, educação pública de adultos e crianças. Tal ação visa a promover a alfabetização nutricional, a implementar impostos efetivos sobre bebidas com açúcar, a adotar medidas eficazes na legislação, restringindo a comercialização de alimentos pouco saudáveis nas escolas e fomentando as campanhas de incentivos, no sentido prioritário de ajudar as pessoas a ingerir alimentos saudáveis e a praticar atividade física regular (WHO, 2018).

Nessa perspectiva, ações de marketing social que incluam os diversos atores envolvidos no consumo alimentar dos jovens se mostram necessárias, na medida em que se propõe uma discussão mais ampla sobre questões alimentares em torno do indivíduo. Dessa forma, é possível fazer uso da abordagem ecológica de marketing social para enfrentar questões mais complexas que a perspectiva do marketing social tradicional não comporta (Birch \& Ventura, 2009). Resumidamente, o modelo ecológico de marketing social fraciona a análise e as intervenções comportamentais em três níveis de influência downstream, midstream e upstream - exigindo que as várias partes interessadas trabalhem juntas em cada um desses níveis para oferecer uma gama de intervenções (Brennan et al., 2016, Dibb \& Carrigan, 2013, Hoek \& Jones, 2011).

Corroborando essa perspectiva de análise, a Organização Mundial de Saúde informa que nenhuma intervenção única pode deter o aumento da obesidade na infância e na adolescência por conta própria, é necessária uma ampla gama de ações em larga escala para que a crescente onda de obesidade seja transformada. Isso exigirá o envolvimento de múltiplos setores, incluindo educação, comunicações, comércio, planejamento urbano, agricultura e saúde (WHO, 2018). Dessa forma, este estudo se torna necessário, na medida em que analisa a atuação e a percepção dos agentes dos níveis upstream e midstream sobre o comportamento de consumo alimentar dos jovens, que tem imposto suas consequências para a saúde pública no longo prazo.

Nesse campo de ação, o foco dessa pesquisa é fundamentado na análise do consumo alimentar e dos hábitos que impactam negativamente a saúde pública, a partir da compreensão dos aspectos de regulação e educação que influenciam o consumo alimentar dos jovens. Alinhado a esse debate, o objetivo desse artigo é analisar o comportamento alimentar dos jovens, na perspectiva dos níveis upstream e midstream de marketing social, uma vez que os atores que ocupam estes níveis estão vinculados no planejamento e execução de políticas públicas de saúde dos municípios. Para tanto, a revisão de literatura discute as dimensões que orientam a ações em torno da promoção da alimentação saudável no âmbito upstream e midstream, abordando as barreiras, os aspectos de regulação saudável e a consciência para saúde desenvolvida pelos jovens. Em termos metodológicos, são apresentados os processos qualitativos que fundamentaram a coleta e análise dos dados. Por fim, são expostos os resultados e as considerações finais, com intuito de discutir os aspectos que influenciam os agentes que desenvolvem a promoção e a adoção de uma alimentação saudável.

\section{Dimensões do consumo alimentar saudável e o modelo ecológico de marketing social}

0 viés de ação do marketing social é gerar um benefício positivo para a sociedade, assim, o entendimento é que o agente ofertante da mudança social deve acreditar que a mudança proposta promoverá em longo prazo o bem-estar social pela redução de algum dos problemas proeminentes da sociedade (Andreasen, 1994, Kotler \& Lee, 2011). Apesar dos agentes de mudança social exercerem um interesse 
permanente no comportamento de mudança no âmbito individual, o escopo das intervenções vem ampliando-se, movendo-se e seus praticantes tornando-se mais estratégicos para combater comportamentos de consumo prejudiciais à sociedade ou para incentivar comportamentos saudáveis. Isso significa mudança de atitudes, comportamentos e crenças para criar mudança social (Dibb, 2014). A partir dessa ampliação, o modelo ecológico de marketing social se desenvolveu e dividiu a atuação de marketing social em três níveis de influência - downstream, midstream e upstream (Dibb, 2014).

As influências sociais e políticas se referem ao nível upstream, ou seja, ao ambiente estrutural que pode influenciar o comportamento (Gordon, 2013), na medida em que reduz as barreiras para a sociedade aderir à mudança de um comportamento desejado, através de intervenções sociais e políticas públicas (Wallack, 2002). A abordagem midstream refere-se ao ambiente social mais imediato, como as organizações e as comunidades que desempenham um papel importante no apoio a comportamentos desejáveis que captem influências sociais no indivíduo alvo a nível comunitário. É possível citar como exemplo o âmbito escolar, em que pode se desenvolver uma mudança de comportamento a longo prazo na educação alimentar (Hastings, 2003). O nível Downstream refere-se a influências sobre o comportamento no âmbito individual, que procuram reduzir os problemas da população através de programas de mudança comportamental voltados para o indivíduo, o que inclui uma configuração dos programas para promover mensagens de práticas saudáveis voltadas para as famílias. (Dibb, 2014, Brennan \& Wood, 2016).

Nesse ímpeto, o problema da má alimentação evidencia um conjunto de perspectivas concorrentes e as vias causais inter-relacionadas (Shelley, 2012). As sugestões para as iniciativas em torno do consumo alimentar saudável podem se concentrar em todos os níveis de atuação, sendo proposto que as intervenções devem ser entregues em um contínuo que varia de down-, mid-, upstream para sustentar a mudança de comportamento. De maneira geral, as formas pelas quais a obesidade é percebida remetem à discussão voltada para o nível upstream, cuja questão se norteia por um viés de regulação e ou política de ação entre o público e o Estado (Campbell, 2012).

É possível defender que ações para redução e prevenção do consumo de alimentos industrializados e pouco saudáveis exigem uma ação institucional formal que estimule e apoie mudanças institucionais informais. Ou seja, uma mudança na cultura alimentar é apoiada por mudanças, como a adição de impostos aos alimentos classificados como pouco saudáveis (aquele que contém altos níveis de açúcar, gordura ou sal). Por outro lado, Domel (2000) afirma que os padrões de consumo de alimentos para crianças são diretamente afetados pelo ambiente familiar e pelo ambiente escolar posicionados em nível midstream. Três fatores ambientais influenciam o consumo de alimentos, como: a cultura e disponibilidade dos alimentos nas escolas, a exposição de mídia a mensagens de alimentos e o acesso a alimentos saudáveis e não saudáveis. Além de ter um efeito direto sobre o excesso de peso e a obesidade na infância, o ambiente sociocultural e físico também influenciam as normas de consumo de alimentos familiares (Davison, 2001).

Para este estudo, as dimensões analisadas buscam compreender o comportamento alimentar dos adolescentes ao saber quais as principais barreiras que dificultam a adesão de hábitos alimentares adequados, avaliar a regulação alimentar brasileira e analisar a consciência sobre saúde desenvolvida pelos jovens a partir da percepção dos agentes upstream e midstream. Um fator que pode ser considerado como uma barreira à alimentação saudável é o fator cultural. Nas últimas décadas, a família mudou muito, desde quando a mulher entrou no mercado de trabalho e, consequentemente, precisou conciliar a vida profissional com as tarefas domésticas. Assim, muitas mudanças ocorreram no ambiente familiar, o que afetou o modo de se alimentar, pois em busca de comodidade e praticidade, os alimentos industrializados passaram a estar cada vez mais presentes (Vaz, 2018).

No que se refere à regulação alimentar no Brasil, Proença (2012) acredita que existe uma grande fragilidade, um exemplo disso são as recomendações nos rótulos dos alimentos relacionadas ao consumo de gordura trans, as quais são muito controversas em termos nutricionais. Não obstante, é necessário reforçar que a rotulagem nutricional não é a única solução. A melhoria dos hábitos alimentares e a reversão do cenário atual possuem causa multifatorial, requerendo a adoção de políticas públicas que modifiquem os fatores de risco, bem como promovam alterações comportamentais no âmbito individual e coletivo dos consumidores (Verstuyf, 2016, Brasil, 2018). Para Martins et al., (2014), uma alternativa é propor a regulação da propaganda de alimentos, já para a Anvisa tal medida impactaria ao mesmo tempo em dois setores poderosos na economia brasileira: a indústria de alimentos 
e a indústria de propaganda.

Em relação à dimensão consciência sobre saúde, a Organização Mundial de Saúde considera que os sistemas alimentares, os padrões de marketing e os estilos de vida pessoais devem evoluir de forma a facilitar a vida mais saudável das pessoas, ao incentivar e favorecer o comércio de alimentos que lhes trazem os maiores benefícios para à saúde, sendo este o principal objetivo das políticas de saúde pública (Apaolazaa, 2018, Who, 2018). Tais processos exigem mudanças de longo prazo no pensamento e na ação nos níveis downstream e midstream, sendo mais provável que a mudança individual seja facilitada se o macroambiente - upstream apoiar as opções disponíveis como saudáveis e recompensadoras.

\section{Método}

Este tópico tem por objetivo descrever os procedimentos metodológicos que foram utilizados na investigação da seguinte questão: qual a percepção dos agentes dos níveis upstream e midstream do modelo ecológico de marketing social sobre o consumo alimentar dos jovens? Desse modo, para analisar os níveis upstream e midstream foi realizada uma pesquisa qualitativa de caráter exploratório, tendo em vista a necessidade de compreender o consumo alimentar saudável entre adolescentes e as ações realizadas em torno de sua promoção (Minayo, 2004).

0 roteiro desenvolvido para as entrevistas foi dividido em dois momentos, no primeiro optou-se por entrevistar agentes no nível upstream - secretários municipais de saúde responsáveis pela formulação de política de saúde do município, e no segundo momento, optou-se por entrevistar agentes midstream - nutricionistas da região, responsáveis pela parte de execução das ações definidas pelos municípios, ligados diretamente com os indivíduos alvos. 0 contexto da pesquisa foram municípios paraibanos e os sujeitos da pesquisa atuam de forma conjunta, ou seja, são secretários de saúde e nutricionistas dos mesmos municípios. Foram elaboradas doze questões divididas em três dimensões: as barreiras para alimentação saudável (Hilger, Loerbrocks \& Diehk, 2017); a regulação alimentar (Verstuyf, 2016), e, a consciência para saúde (Apaolazaa, 2018).

A pesquisa foi realizada com dez indivíduos, sendo cinco agentes upstream (as entrevistas são apresentadas com os códigos: E1, E3, E4, E6 e E7), com formação profissional nas áreas de Enfermagem, Odontologia, Educação Física e História; e cinco agentes midstream (E2, E5, E8, E9, E10), sendo todos formados em Nutrição. As entrevistas tiveram início no dia 24/05/2018 e foram encerradas no dia 21/06/2018, e as gravações de áudio têm duração de 179 minutos e 68 segundos. Foram obtidos um total de 88 laudas de transcrição, configuradas em página A4, com fonte Times New Roman, tamanho 12 e espaçamento simples. Para a análise dos dados, fez-se o uso da análise de conteúdo, sendo adotadas como orientação as três dimensões definidas no roteiro (Flick, 2009).

\section{Análise dos resultados}

Neste tópico são apresentados os resultados oriundos da análise de conteúdo qualitativa procedida. As dimensões abordam as barreiras para a alimentação saudável, a regulação alimentar e a consciência sobre a saúde dos adolescentes a partir da percepção dos agentes dos níveis upstream e midstream.

\subsection{Barreiras para a alimentação saudável}

A discussão se inicia a partir do nível upstream, ao analisar a percepção dos secretários de saúde municipais em relação ao que consideram como barreira para os jovens aderirem a uma alimentação saudável. Foi identificado que muitos dos entrevistados consideraram a questão midiática, no sentido de induzir o jovem a ser alvo do consumismo desordenado. 0 que está de acordo com Davison (2001), que aponta como fatores ambientais capazes de influenciar o consumo de alimentos por jovens e crianças: a disponibilidade alimentar nas escolas, a exposição de mídia a mensagens de alimentos e o acesso da vizinhança a alimentos saudáveis e não saudáveis.

"[...] a pressão que o jovem sofre perante a mídia, eu acho que isso acaba dificultando eles perceberem o que é uma alimentação saudável. " (E7)

"[...] existe uma dificuldade enorme para estar proporcionando uma melhor qualidade de vida a essa população alvo, diante do consumismo." (E1)

Outro fator de influência é a questão cultural. Com o passar dos anos, foi identificado que os hábitos alimentares se transformaram e os alimentos passaram a ser mais instantâneos, ou seja, o hábito de 
preparar alimento foi substituído por comidas prontas, congeladas e de rápido preparo. Ou seja, o consumo alimentar absorveu o imediatismo e a conveniência próprios da sociedade contemporânea, como apontam os relatos dos gestores públicos.

"A facilidade e a praticidade dos industrializados pelas mães que hoje trabalham muito, e também acho que a questão cultural das mães é o fator principal na minha opinião. " (E6)

"A maior dificuldade que tem hoje é a questão dos Fast food [...], uma porta de entrada, para toda e qualquer patologia, o jovem não se preocupa com isso, então ele quer na hora do lanche comer sua a "pipoquinha" seu refrigerante". (E3)

Outro ponto que deve ser levado em consideração é a fragilidade de apoio da esfera pública, no que diz respeito a incentivos sociais, políticos e financeiros para esse público-alvo. Campbell (2012) aponta que é necessária uma atuação mais incisiva do Estado na regulação e nas políticas públicas de combate à obesidade, entretanto o que se percebeu foi que o governo não tem dado atenção devida a esse tópico e pouco tem estimulado a adoção de boas práticas e conscientização do jovem, embora os gestores públicos reconheçam que a inclusão de nutricionistas na alimentação escolar pode ser considerada um aspecto favorável.

"Existe apoio, mas muito deficiente, até mesmo porque a gente não tem um recurso destinado para isso" (E1)

"Hoje é muito interessante todas as escolas já terem nutricionistas, então assim, isso já é um ponto muito positivo, [...] então, a lógica e a tendência é que se tenha de fato uma alimentação saudável para esse jovem, seja na faixa etária do ensino fundamental ou médio [...]" (E7)

Kilbourne, Mcdonagh \& Prothero (1997) entendem que ações para redução e prevenção da obesidade exigem uma forma institucional formal que estimule e apoie mudanças institucionais informais. Ou seja, uma mudança na cultura alimentar é apoiada por mudanças institucionais formais, como a adição de impostos aos alimentos classificados como pouco saudáveis (aquele que contém altos níveis de açúcar, gordura ou sal). No contexto analisado, as informações são ofertadas nas Unidades Básicas de Saúde e também nas escolas, os nutricionistas vão até os alunos e orientam, de maneira didática, alcançando principalmente os alunos da educação básica. Os secretários de saúde apontam que este é um dos programas que viabilizam esse acesso a priori, trabalhando a questão mais educativa.

"Através das Unidades Básicas de Saúde com as enfermeiras, tal... Fazendo palestras, através do NASF também. " (E4)

"A gente tem esses programas né? Do Núcleo Ampliado da Saúde da Família, nós temos o programa Saúde na Escola que é do Ministério da Saúde em parceria com o Ministério da Educação, onde a gente leva todos os nossos profissionais da área da saúde para as escolas, aí é onde a gente pega aquela educação básica e o fundamental I que é show de bola, tudo que você passa eles chegam em casa e transmitem para os pais, é impressionante. "(E3)

É importante desenvolver medidas que o jovem se sinta atraído a desenvolver uma busca ativa por informações alimentares, para que consigam enxergar a importância de ter uma alimentação saudável e aderir à prática de forma duradoura. Os gestores refletem que o modo como a informação chega ao público-alvo pode influenciar os resultados, então, deve-se buscar repassar da melhor maneira possível, para que os sujeitos interpretem a informação como um aspecto do seu estilo de vida.

"Acredito que seria um trabalho intersetorial, onde todos falassem a mesma língua destinada ao jovem para que ele realmente sentisse que aquilo é necessário. " (E1)

Por outro lado, no nível midstream foi possível identificar, a partir de entrevistas com nutricionistas da região vinculados ao setor público, quais as principais barreiras para o jovem aderir a uma alimentação saudável. Assim como apontado pelo nível usptream, emergiram as questões midiáticas e culturais, conforme Davison (2001) colocou.

"Eu acho assim que a mídia ajuda muito a dificultar essa a questão da alimentação saudável, porque se você ligar a tevê vai ter muitas propagandas de comidas que não faz bem para saúde né? Então isso chama atenção dos jovens. "(E2).

"[...] tem muitas influencers que tem uma rotina de vida saudável que transparece essa rotina de vida saudável e passa através das redes sociais, o jovem assiste tudo isso, mas eles ficam ali divididos entre seguir um caminho e entre seguir outro caminho [...]" (E8).

E como já foi discutido na análise upstream, com o passar dos anos as famílias têm-se adequado a outra cultura, principalmente depois que as mulheres ganharam espaço no mercado de trabalho. A partir daí, a família foi aos poucos perdendo o hábito de se alimentar em casa, refletindo-se na vida dos 
jovens, que aprendem desde cedo a trocar alimentação saudável por alimentos de rápido preparo. Outro indicador que foi considerado como barreira para a alimentação saudável foi o fator tempo. Nos relatos dos agentes midstream é evidente que a falta de tempo tem prejudicado a alimentação dos jovens, assim como aspectos de formação no preparo de alimentos.

"[...] essa cultura está vindo mais de casa, às vezes em casa os pais não comem frutas, não comem verdura, então isso tudo reflete na alimentação da criança." (E5).

"[...] as vezes chego no consultório querendo mudar o hábito, mas aí a gente vê que o hábito da família já é um hábito desregrado, e aí o jovem acaba acompanhando." (E10).

"Então assim, o pessoal precisa muito principalmente os jovens, saber direitinho como é a forma de se alimentar mesmo sem ter tempo [...] é isso que falta, é o horário, organização e o próprio incentivo de uma alimentação saudável" (E9).

Os agentes levantaram a proposta de investir em uma ação direcionada para o público, não apenas criando um conceito de acesso à informação, mas tornando-a atrativa para que o jovem possa aplicá-las e desenvolvê-las no seu cotidiano alimentar. Os entrevistados refletiram sobre a necessidade de desmitificar o conceito de alimentação saudável. Ademais, os nutricionistas evidenciaram a inadequação da alimentação oferecida pelas escolas, uma vez que poucos são os recursos destinados para a compra de alimentos de maior valor nutricional.

"acho que por ser jovem tinha que criar um mecanismo jovem de passar para eles a informação de uma forma que eles venham a entender e aceitar [...] de forma que eles entendessem que a má alimentação vai causar um distúrbio a longo prazo [...]porque o jovem de hoje eles veem alimentação saudável como uma coisa ruim, que não tem gosto. "(E8).

"[...] o recurso dado para alimentação é muito pequeno, então acaba que você tem que baratear alimentação e acaba fugindo um pouco do que seria uma boa alimentação [...]” (E10).

De maneira geral, as barreiras para a alimentação saudável indicam que as políticas públicas em torno da alimentação devem priorizar o ambiente escolar, seja da iniciativa pública, seja da privada, uma vez que muitos adolescentes não possuem um ambiente familiar com controle alimentar adequado. A partir de então, o público que recepciona as ações educacionais pode desenvolver uma capacidade reflexiva em torno do alimento, aplicando em seu ambiente familiar o que aprendeu e, principalmente, o que vive no ambiente escolar. Ademais, a participação dos pais no ambiente escolar também reforçaria a mudança comportamental e a consolidação de uma política pública em torno da alimentação saudável.

\subsection{Regulação alimentar}

Nesse item, os agentes upstream explanaram sua percepção em relação à regulação alimentar, sendo discutidas e avaliadas as políticas de saúde do município em torno da alimentação saudável. 0 que se percebeu foi que a regulação alimentar no Brasil ainda é muito deficiente, diante de tantos recursos, os gestores apontam para a necessidade de ampliar os programas que já existem para conseguir uma mudança de comportamento alimentar desejável e para sdiminuir os índices de DCNT que só aumentaram nos últimos anos. Os entrevistados ainda relataram que não há incentivos do governo direcionados a esse público jovem, existem políticas públicas voltadas para o público infantil e para o idoso, implicando uma lacuna entre esses dois públicos que precisa ser reavaliada.

"Muito deficiente, tanto que a gente tem aí pessoas obesas e desnutridas, temos pessoas magrinhas e ao mesmo tempo obesas com colesterolemia e outras infinidades de fatores que podem proporcionar doenças." (E1).

"[...] para o jovem não tem um programa voltado, para que haja um incentivo na questão de melhoria na alimentação". (E4).

"[...] normalmente é trabalhado no município com crianças e idosos por conta das políticas públicas, o adolescente, por exemplo, nas escolas onde tem o ensino médio né no caso, as nutricionistas quase não têm acesso, a gente não tem trabalhado em cima deles. "(E6).

Os resultados mostraram que os agentes estão conscientes de que as políticas públicas ainda não são suficientes e julgam importantes que a regulação alimentar seja mais efetiva no Brasil. Proença (2012) acredita que existe uma grande fragilidade na regulação, um exemplo disso são as informações nos rótulos dos alimentos, como aquelas relacionadas ao consumo de gordura trans.

"Tem que ter a questão dessa regulação, para que aja um direcionamento do jovem, muitas vezes a informação não chega até o jovem para que ele tenha o discernimento de qual alimento ele deve escolher, então assim tem que haver essa regulação, tem que ter um norte pra isso." (E4) 
Corroborando a percepção dos gestores públicos, a regulação na ótica dos nutricionistas traz uma fragilidade de investimento em políticas de saúde, na medida em que indicam uma atuação restrita por parte dos órgãos reguladores que já existem. 0 rótulo emerge como uma das exigências alimentares que são cumpridas, entretanto os profissionais reconhecem as dificuldades de compreensão dos consumidores e também a falta de fiscalização das informações contidas na rotulagem dos produtos. Os relatos apontam tais percepções.

"Único procedimento aqui no Brasil da regulação alimentar é o rótulo, por exemplo, eu vou lá na embalagem e uma das coisas que eles são obrigados por lei a colocar na embalagem esse contém ou não glúten, isso é obrigado! [...]E se essa pessoa leiga de repente tem uma intolerância ao glúten e não sabe o que é isso? O que vai adiantar ter no rótulo "contém glúten ou não contém glúten?" (E8)

"a gente vem muitos erros nos rótulos, mentiras mesmo, na frente do produto as vezes tem zero açúcar e a gente nutricionista sabe quando vai olhar tem um outro nome no lugar do açúcar". (E10)

Com isso, pode-se ver que a regulação alimentar no Brasil não é eficiente, embora existam algumas normas, estas não são fortes o bastante para impedir as infrações na comercialização. É preciso pôr em evidência tais normas para que todos tenham conhecimento, e consigam compreendê-las e fiscalizá-las no âmbito individual. Como dito pelo entrevistado E9.

"[...] deveria ter mais leis de proibição [...] uma fiscalização mais aprofundada, uma fiscalização grande mesmo, para que evitasse esse tipo de coisa [...]" (E9)

Percebeu-se a partir dos relatos dos nutricionistas que as pessoas ainda são bastante leigas no assunto alimentação, entendem pouco e muitas vezes precisam de orientação básica daqueles que detêm conhecimento especializado. 0 entrevistado (E2) ressalta a importância da informação e da formação em torno da alimentação, mostrando para as pessoas que a má alimentação pode resultar em doenças como diabetes e hipertensão.

"se essas campanhas fossem trabalhadas na internet, com certa insistência, vai chegar um momento que os jovens vão atentar para aquilo ali [...], e a partir do momento que a pessoa tem acesso à informação ela com certeza vai abrir os olhos para muitas outras coisas." (E8).

Foi posto em discussão, durante as entrevistas entre os níveis upstream e midstream, algumas ações existentes no município, inclusive umas delas já executadas. As iniciativas do governo são vistas como boas, ao desenvolver uma proposta positiva e atingir um número de pessoas considerável, porém ainda muito frágil. É preciso melhorar o apoio e a ampliação de programas voltados para essa temática para se tornarem mais efetivas, como o entrevistado (E5) relata "as políticas de saúde no papel são lindas, agora é uma grande dificuldade de se colocar em prática".

"[...] a gente está no NASF, não tem como colocar em prática todas as políticas de saúde, o que é que a gente faz aqui? A gente faz o PSE (Programa de Saúde na Escola), o SISVAN (Sistema de Vigilância Alimentar e Nutricional), e agora estou entrando no NUTRISUS." (E5).

"As ações acontecem muito mais no PSF, $e$

ntão depende muito do corpo do NASF, de como os nutricionistas trabalham em relação a isso, mas não sai de dentro do NASF, quer dizer não sai de dentro dos PSF, aquele trabalho não é externado, então os jovens que teriam que ir até o PSF para ter essa informação, então o trabalho municipal também não é convincente, não satisfaz." (E8).

"[...] tem também a Política Nacional de segurança alimentar que é para garantir o direito que todos têm alimentação, mas que eu vejo que é muito papel e pouca ação, não é muito, eu não vejo sendo bem executado". (E10).

Em suma, embora os agentes reconheçam a necessidade de um aprimoramento nas políticas públicas em torno da alimentação saudável para os adolescentes e jovens, há um evidente descompasso entre as propostas dessas políticas e as ações desenvolvidas em nível de município. Há um excesso de sistemas e programas mencionados pelos gestores, e que são vistos pelos nutricionistas como incapazes de alcançar o público jovem, e muitas das ações desenvolvidas são sempre no escopo de feiras de saúde e de ações pontuais sobre as DCNT. Além disso, é evidente que os agentes do nível midstream desempenham funções técnicas especializadas, com dificuldade de compreensão de quais elementos poderiam ser empregados para o convencimento e desenvolvimento de um comportamento favorável à alimentação saudável pelo público em questão. Muitos dos entrevistados apontaram o acesso à informação como sendo um dos principais fatores de mudança comportamental, desconsiderando a influência da família e o ambiente social em que o sujeito está inserido. 


\subsection{Consciência sobre saúde}

Nesta dimensão foi avaliado se a consciência sobre os aspectos de saúde e a alimentação influenciam os jovens a desenvolver um estilo de vida saudável. A princípio, os entrevistados analisaram que as pessoas podem ser mais conscientes ao ponto de tomar decisões que mudem seu estilo de vida a partir do comportamento de outras pessoas que já possuem um estilo de vida saudável. A fala converge com tal constatação.

"desde que eu queira absorver o que essas pessoas saudáveis estão dizendo não é? Porque assim, é difícil não é fácil, por exemplo, se eu for falar de alimentação saudável em certo momento alguém vai ouvir, mas vai olhar para o meu corpo e vai dizer como é que esse cara está falando de alimentação saudável se ele está acima do peso? É como eu digo, a gente é muito pelo aquilo que vê né?" (E3). "Acredito, acredito que o ambiente provoca mudança[...] se eu convivo com uma pessoa que faz uma alimentação certinha, tem os horários regulados que me explica o porquê está comendo aquilo, qual o benefício, eu acredito que tenha um resultado positivo. "(E10)

Mediante tal entendimento, foi debatido como o governo municipal avalia a necessidade de contribuir para uma alimentação saudável entre os jovens. Segundo relato do entrevistado (E2) o primeiro passo seria obedecer aos programas que são disponibilizados, por exemplo, o Programa da Agricultura Familiar que foi criado com o intuito de fazer com que pelo menos $30 \%$ da alimentação fosse baseada em produtos oriundos desse meio. Por sua vez, os indivíduos entendem que o desenvolvimento de uma consciência alimentar deve ser considerado uma questão de saúde pública, pois como o entrevistado (E6) relata "é uma prevenção de doenças". Os relatos abaixo confirmam tal entendimento.

"Alimentação saudável é igual a uma vida saudável, se você não tem uma alimentação saudável, vai começar a parecer problemas cardíacos problema respiratório, dor de cabeça, essas coisas..." (E4)

"[...] a alimentação salva qualquer geração, seja de jovens, seja de idosos, eu acho que o hábito de se alimentar bem e saudável, é sem dúvidas essencial. " (E7)

Outro fator que se sobressai nas falas dos agentes é a importância de conscientizar a população e trabalhar a prevenção das doenças (Hoek \& Jones, 2011), alinhado ao pensamento da entrevistada (E6) que acredita que o município não é efetivo pela quantidade de medicamentos que oferece, mas se torna efetivo a partir do momento que consegue atingir uma redução desse número. A entrevistada frisa a importância de se trabalhar a prevenção, investir mais na atenção básica do que na medicina de média e alta complexidade, com o intuito de reduzir o quadro de adoecimento da população.

"Seria interessante que a gente trabalhasse a prevenção, para não ter que estar pagando tão caro por isso, em um país onde a saúde não tem recursos para ser custeado, os recursos são mínimos". (E1)

A partir da percepção dos agentes midstream foi possível analisar o comportamento alimentar dos jovens atualmente, e diante dos relatos, os nutricionistas consideraram o comportamento dos jovens como não adequado. Muitos nutricionistas consideraram que a alimentação dos filhos é uma responsabilidade dos pais, logo se o filho tem uma alimentação desregrada é porque foi alvo da permissividade familiar, como coloca o entrevistado (E10).

"Não há uma consciência do que se está fazendo, é muito de ir pelo momento, de querer alcançar um objetivo muito rápido, e não parar para pensar até que ponto isso vai me fazer mal a longo prazo, o jovem ele quer uma coisa muito imediata. [...] eu atendo crianças que a mãe chega para mim achando que eu que vou botar o filho no lugar, e aí eu tenho que explicar que a minha parte vou fazer, mas em casa o pai a mãe é que compram [...] panham demais a mesma sequência, a mesma rotina dos pais, e aí a gente vê muito filhos de pais diabéticos se tornando também diabéticos, filhos de pais hipertenso se tornando também já com início de pressão alta, com obesidade. "(E10)

Ainda foi analisada a percepção dos agentes midstream sobre como os hábitos alimentares dos jovens poderiam ser melhorados. Os entrevistados acreditam fortemente que essa mudança pode acontecer a partir de uma reeducação alimentar e de uma reestruturação dos hábitos familiares. No sentido mais amplo, um dos entrevistados entende que é preciso relacionar a atividade física com a reeducação alimentar para que os resultados em termos de saúde preventiva sejam sobressalentes.

"o importante hoje em dia é a reeducação alimentar, uma reeducação e uma orientação nutricional, ela é mais eficaz do que uma dieta no papel, ela é bem mais eficaz, por que você vai saber o que pode e o que não pode." (E9). "Algumas pessoas que pegam gosto pela atividade física e em paralelo vão para uma alimentação mais saudável, porque aí ver que uma coisa depende da outra, eu até acho que ultimamente tem se aumentado essa consciência [...] e desmistificar aqui alimentação saudável é muito cara, que não e desmistificar isso, falta muita coisa ainda eu acho." (E10). 
Sendo assim, para que haja uma mudança de comportamento alimentar desejável, é necessário que o indivíduo alvo possa ser informado da maneira correta, no sentido de estar consciente dos benefícios da adoção de uma vida saudável, e, consequentemente, se sinta motivado a fazer essa busca ativa por meio dos programas sociais desenvolvidos. De maneira geral, podemos afirmar que há uma convergência na percepção dos entrevistados com relação à consciência para a saúde dos jovens, na medida em que acreditam que a pressão social (dos familiares e do ambiente escolar) favorece sobremaneira a adoção da alimentação saudável, além de enfatizar a necessidade de uma educação alimentar como política de saúde para a promoção da mudança comportamental em longo prazo.

\section{Considerações finais}

O presente estudo identificou que, a partir da discussão dos agentes upstream e midstream, as principais barreiras para os jovens aderirem a uma alimentação saudável se relacionam à questão midiática, que se faz presente de forma muito efetiva na sociedade em geral, por meio dos canais de massa (redes sociais, televisão) e que atua no sentido de induzir ao jovem a ser alvo do consumismo desordenado; e, à questão cultural que atinge o contexto alimentar das famílias e tem se transformado ao longo dos tempos. Tais achados são corroborados pelo estudo de Davison (2001), que aponta como o fator ambiental de influência a exposição das mídias e a forma como a rotina dos lares tem se transformado.

Outro indicador que foi considerado como barreira para uma alimentação saudável foi o fator tempo. Os resultados apontaram que os indivíduos procuram sempre se alimentar do que é mais prático e rápido e que a falta de organização do tempo tornou a sociedade dependente dos alimentos industrializados. 0 estudo mostrou a fragilidade de apoio direcionado a essa temática, ressaltando a deficiência de iniciativa do governo, que mesmo diante de um número crescente de Doenças Crônicas Não Transmissíveis no mundo, pouco tem feito para combater estas doenças, desencadeadas principalmente pela alimentação não adequada.

Os resultados mostraram que a regulação alimentar no Brasil ainda é muito deficiente, existem raras medidas de regulação, poucas leis, e o investimento ainda é mínimo em políticas públicas e de saúde para o jovem, apesar de existirem algumas ações direcionadas ao público infantil e aos idosos. Por sua vez, o estudo demonstrou o interesse que os agentes têm em melhorar a alimentação dos jovens, ao considerarem ser necessário investir em educação e reeducação alimentar, a partir de políticas públicas mais efetivas, ampliando os programas e tornando-os mais didáticos e atrativos. Os entrevistados acreditam que o convívio com pessoas que já possuem um comportamento alimentar saudável permite uma disseminação mais facilitada sobre os benefícios para a saúde, assim como desmitifica questões como a falta de sabor dos alimentos saudáveis. Isto reforça o achado de que os hábitos alimentares inadequados dos jovens são influências da permissividade familiar, considerando que os pais são os responsáveis por moldar o paladar dos filhos quando crianças.

\subsection{Limitações da pesquisa}

As principais limitações da pesquisa se relacionam à falta de disponibilidade dos secretários de saúde e dos nutricionistas. Ademais, a pesquisa em si pode alcançar mais munícipios do estado da Paraíba para uma análise mais completa em termos de atuação das políticas públicas para a alimentação.

Por fim, a proposta do estudo foi compreender aspectos de um público jovem e adolescente, no entanto torna-se prioritário analisar ações que estejam sendo desenvolvidas para o público infantil, dado o seu estado inicial de formação como ser humano e as fragilidades características dessa faixa etária.

\subsection{Sugestões teóricas e práticas}

Como implicação prática e teórica, espera-se que esta pesquisa contribua para a geração de ações de marketing social mais eficientes, na medida em que essas ações possam reforçar o desenvolvimento de políticas públicas que explorem os benefícios de uma alimentação saudável e elaborem incentivos para a adoção dessa rotina alimentar. Conforme os resultados, as ações de intervenção de marketing social devem priorizar campanhas direcionadas para as comunidades locais, desenvolvendo abordagens de educação alimentar e de habilidades de cozinha entre as famílias e os jovens no ambiente escolar. Os resultados apontam ainda para a necessidade de incentivar maior autonomia nas escolhas alimentares, 
o que pode ser feito a partir da prestação de orientação dos profissionais de saúde nas feiras de alimentos da cidade e nas feiras de saúde promovidas pelos municípios.

Com vistas a suplantar as dificuldades da gestão pública em relação à alimentação saudável, as atividades de marketing social desempenhadas por organizações sociais devem explorar o estilo de vida saudável, caracterizado por atividades físicas regulares e por uma alimentação adequada aos padrões estabelecidos pelo guia alimentar da Organização Mundial de Saúde; pela propagação de receitas, de produtos minimamente industrializados; e pelo incentivo ao consumo de produtos naturais e dietas plant based. Concomitantemente, uso das redes sociais e de personalidades da Internet são uma das ferramentas contemporâneas adequadas para a divulgação de um estilo de vida saudável, visando a construção de uma percepção que a alimentação saudável é conveniente e facilmente incluída no dia a dia das pessoas. Além disso, tais instrumentos de comunicação parecem ser eficientes para explicar os componentes dos alimentos, reduzindo a necessidade de capacidade técnica para o entendimento de informações alimentares básicas.

Ademais, as campanhas de marketing social em torno da alimentação saudável devem também discutir as ações de marketing do setor alimentício, trazendo uma perspectiva mais crítica aos consumidores, uma vez que os agentes identificaram a mídia como sendo um dos influenciadores da má alimentação. Nessa esfera, os rótulos e as informações nutricionais dos alimentos são reconhecidos pela dificuldade de interpretação do público, tornando-o vulnerável diante da diversidade de ofertas alimentares. É possível que os atores e as entidades defensoras dos interesses dos consumidores atuem para exigir e propor uma regulação da propaganda alimentar, além da criação de impostos e outras formas de punição nos diferentes níveis de gestão pública.

Por fim, sugere-se aos gestores o desenvolvimento de decretos e leis que possam aprimorar a rotina alimentar do público aqui analisado, de modo a resultar em qualidade de vida para os adolescentes. É sugerido para uma análise mais completa, que sejam realizados estudos com essa iniciativa de promoção de saúde pública sob o escopo de marketing, com agentes downstream, para que seja feito um cruzamento de informações dos três níveis - up- mid e down.

\section{Referências}

Abarca-Gómez, Leandra et al. (2017). Worldwide trends in body-mass index, underweight, overweight, and obesity from 1975 to 2016: a pooled analysis of 2416 population-based measurement studies in 128. 9 million children, adolescents, and adults. The Lancet, 390(10113), 2627-2642.

Andreasen, A. R. (1994). Social marketing: Its definition and domain. Journal of public policy \& marketing, 13(1), 108-114.

Apaolaza, V., Hartmann, P., D'Souza, C., \& López, C. M. (2018). Eat organic-Feel good? The relationship between organic food consumption, health concern and subjective wellbeing. Food Quality and Preference, 63, 51-62.

Birch, L. L., \& Ventura, A. K. (2009). Preventing childhood obesity: what works?. International journal of obesity, 33(S1), S74.

Brasil. Anvisa. (2018). Relatório Preliminar de Análise de Impacto Regulatório sobre Rotulagem Nutricional. Brasília.

Brasil. MDS. Caisan. (2018). Plano Nacional de Segurança Alimentar e Nutricional. PLANSAN 2016-2019. Brasília.

Brennan, L., Previte, J., \& Fry, M. L. (2016). Social marketing's consumer myopia: applying a behavioural ecological model to address wicked problems. Journal of Social Marketing, 6(3), 219-239.

Campbell, A. T. (2012). The context for government regulation of obesity around the globe: implications for global policy action. World Medical \& Health Policy, 4(2), 1-48.

Davison, K. K., \& Birch, L. L. (2001). Childhood overweight: a contextual model and recommendations for future research. Obesity reviews, 2(3), 159-171.

Dibb, S. (2014). Up, up and away: social marketing breaks free. Journal of Marketing Management, 30(1112), 1159-1185.

Dibb, S., \& Carrigan, M. (2013). Social marketing transformed: Kotler, Polonsky and Hastings reflect on social marketing in a period of social change. European Journal of Marketing, 47(9), 1376-1398.

Domel, S. B., Baranowski, T., Davis, H., Thompson, W. O., Leonard, S. B., Riley, P., ... \& Smyth, M. (1993). Development and evaluation of a school intervention to increase fruit and vegetable consumption 
among 4th and 5th grade students. Journal of Nutrition Education, 25(6), 345-349.

Flick, U. (2008). Introdução à pesquisa qualitativa-3. Artmed editora.

Gordon, R. (2012). Re-thinking and re-tooling the social marketing mix. Australasian Marketing Journal (AMJ), 20(2), 122-126.

Gordon, R. (2013). Unlocking the potential of upstream social marketing. European Journal of Marketing, 47(9), 1525-1547.

Hilger, J., Loerbroks, A., \& Diehl, K. (2017). Eating behaviour of university students in Germany: Dietary intake, barriers to healthy eating and changes in eating behaviour since the time of matriculation. $A p$ petite, 109, 100-107.

Hastings, G., \& Saren, M. (2003). The critical contribution of social marketing: theory and application. Marketing theory, 3(3), 305-322.

Hoek, J., \& Jones, S. C. (2011). Regulation, public health and social marketing: a behaviour change trinity. Journal of Social Marketing, 1(1), 32-44.

Kilbourne, W., McDonagh, P., \& Prothero, A. (1997). Sustainable consumption and the quality of life: A macromarketing challenge to the dominant social paradigm. Journal of macromarketing, 17(1), 4-24.

Kotler, P. \& Lee, N. (2011). Marketing Social: influenciando comportamentos para o bem. Bookman Editora.

Malta, D. C., Bernal, R. T. I., Lima, M. G., Araújo, S. S. C. D., Silva, M. M. A. D., Freitas, M. I. D. F., \& Barros, M. B. D. A. (2017). Doenças crônicas não transmissíveis e a utilização de serviços de saúde: análise da Pesquisa Nacional de Saúde no Brasil. Rev. Saúde Pública, 51(suppl 1).

Martins, A. P. B. (2014). Publicidade de alimentos não saudáveis: os entraves e as perspectivas de regulação no Brasil. In Publicidade de alimentos não saudáveis: os entraves e as perspectivas de regulação no Brasil.

Proença, R. P. D. C., \& Silveira, B. M. (2012). Recomendações de ingestão e rotulagem de gordura trans em alimentos industrializados brasileiros: análise de documentos oficiais. Revista de Saúde Pública, 46, 923-928.

Minayo, M. C. D. S., \& de Souza, C. (2004). 0 desafio do conhecimento: pesquisa qualitativa em saúde. $8^{a}$ Edição. São Paulo: Hucitec, 201-219.

Shelley, J. J. (2012). Addressing the policy cacophony does not require more evidence: an argument for reframing obesity as caloric overconsumption. BMC public health, 12(1), 1042.

Vaz, D. S. S., \& Bennemann, R. M. (2018). Comportamento alimentar e hábito alimentar: uma revisão. Revista UNINGÁ Review, 20(1).

Verstuyf, J., Vansteenkiste, M., Soetens, B., \& Soenens, B. (2016). Motivational dynamics underlying eating regulation in young and adult female dieters: relationships with healthy eating behaviours and disordered eating symptoms. Psychology \& health, 31(6), 711-729.

Wallack, L. (2002). Public health, social change, and media advocacy. Social Marketing Quarterly, 8(2), 25-31.

Wood, M. (2016). Social marketing for social change. Social Marketing Quarterly, 22(2), 107-118.

World Health Organization. (2018). World health statistics 2018: monitoring health for the SDGs, sustainable development goals. 